\title{
Hyponatremia predicts mortality after stroke
}

\section{Authors}

Roy L Soiza ${ }^{\mathrm{a}, \mathrm{b}}$, Kirsten Cumming ${ }^{\mathrm{b}}$, Allan B Clark ${ }^{\mathrm{c}}$, Joao H Bettencourt-Da Silva ${ }^{\mathrm{d}}$, Anthony K Metcalf ${ }^{\mathrm{c}, \mathrm{d}}$, Kristian M Bowles ${ }^{\mathrm{c}, \mathrm{d}}$, John F Potter ${ }^{\mathrm{c}, \mathrm{d}}$, Phyo K Myint ${ }^{\mathrm{a}, \mathrm{b}, \mathrm{c}, \mathrm{d}}$

\section{Affiliations}

${ }^{a}$ Department of Geriatric Medicine, Aberdeen Royal Infirmary, Aberdeen, Scotland, UK;

${ }^{\mathrm{b}}$ School of Medicine \& Dentistry, University of Aberdeen, Aberdeen, Scotland, UK;

${ }^{\mathrm{c}}$ Norwich Medical School, University of East Anglia, Norwich, Norfolk, UK;

${ }^{\mathrm{d}}$ Stroke Research Group, Norfolk \& Norwich University Hospital, Norwich, Norfolk, UK.

Corresponding author: Dr Roy L Soiza, Wards 303/4, Aberdeen Royal Infirmary, Aberdeen, AB25 2ZN; tel: +441224 558109; fax: +441224 556339; E-mail: roy.soiza@nhs.net

Key words: sodium; dysnatremia; hyponatremia; stroke; mortality; prognosis; outcomes

\section{Word count:}

\section{List of tables:}

Table 1: Sample characteristics categorised by natremic state;

Table 2: Odds Ratios for outcomes using normonatremia as the reference category;

Table 3: Fully-adjusted sub-group analysis by age categories. 


\section{Abstract}

Background: Hyponatremia, the commonest electrolyte imbalance encountered in clinical practice, is associated with adverse outcomes. Despite this, understanding of the association between hyponatremia and stroke mortality outcome is limited.

Aims: To investigate the association between admission serum sodium and mortality at various time-points after stroke.

Methods: Cases of acute stroke admitted to Norfolk and Norwich University Hospital consecutively from January 2003 until June 2013 were included, with mortality outcomes ascertained until the end of December 2013. Odds ratios (OR) or Hazards ratio (HR) for death were constructed for various time points (within 7 days, 8-30 days, within 1 year and over full follow-up).

Results: 8540 participants were included (47.4\% male, mean age $77.3( \pm 12.0)$ years). Point prevalence of hypernatremia and hyponatremia were $3.3 \%$ and $13.8 \%$, respectively. In fully adjusted models controlling for age, sex, pre-stroke modified Rankin score, stroke type, Oxford community stroke project class and laboratory biochemical and hematological results, the ORs (up to one year)/HRs (for full follow up) for the above time points were $1.00,1.11,1.03,1.05$ for mild hyponatremia, 1.97, 0.78, 1.11, 1.2 for moderate hyponatremia, 3.31, $1.57,2.45,1.67$ for severe hyponatremia and $0.47,1.23,1.30,1.10$ for hypernatremia. When stratified by agegroups, outcomes were poorer in younger hyponatremic patients (aged <75 years).

Conclusions: Hyponatremia is prevalent in acute stroke admissions and is independently associated with higher mortality in patients $<75$ years. 


\section{Introduction}

Hyponatremia (serum sodium $<135 \mathrm{mmol} / \mathrm{L}$ ), even when mild, is associated with increased mortality [1-3]. It is the commonest electrolyte abnormality encountered in the general population [4] and in patients with stroke [5]. Furthermore, low sodium is a recognised risk factor for stroke even at levels normally regarded as normonatremic [6]. Moreover, in a study of first ever stroke in patients with chronic kidney disease, hyponatremia was an independent predictor of mortality [7].

Despite this, only two published studies have specifically investigated the association between admission hyponatremia and outcomes after stroke. Hyponatremia was reportedly a predictor of 3-year mortality in acute first-ever ischemic stroke independent of other prognostic predictors [8]. More recently, hyponatremia was associated with higher mortality in hospital, at 3-months and 12-months follow acute stroke [9]. However, it is unclear whether prognosis following acute stroke is affected by the severity of hyponatremia and whether poor outcome persists in patients with mild hyponatremia ( $\mathrm{Na} \mathrm{130-134} \mathrm{mmol/L).} \mathrm{Additionally,} \mathrm{hypernatremia} \mathrm{(Na}$ $>145 \mathrm{mmol} / \mathrm{L}$ ) has been reported to be associated with early neurological worsening following stroke [10].

\section{Aims}

This study investigated the association between admission serum sodium and mortality at various time-points after stroke. Since hyponatremia in older patients is especially common and particularly challenging to manage [12], we looked for differences in the impact of dysnatraemias in older versus younger patients, hypothesising that the prognostic value of serum sodium would vary with age. 


\section{Methods}

We used the Norfolk and Norwich Stroke Register that prospectively recorded consecutive patients with a diagnosis of acute stroke admitted to Norfolk and Norwich University Hospital since 1996. The hospital hosts the only acute stroke unit for a population of approximately 750,000 inhabitants in the city of Norwich and surrounding rural areas. Data collection methods of the register have been previously reported [13]. Briefly, data were obtained from paper-based and electronic records, reviewed and entered onto the register database by the hospital stroke data team. All participants were reviewed and diagnosed by a specialist stroke team. Ethical approval was obtained from the Newcastle and Tyneside National Health Service (NHS) Research Ethics Committee (12/NE/0170) and the study protocol was approved by the Steering Committee of the Norfolk and Norwich Stroke Register.

For the current study, prospectively collected data included basic demographic characteristics, pre-stroke modified Rankin score (mRS) [14], stroke type using the Oxfordshire Community Stroke Project (OCSP) classification [15], co-morbidities and results of hematological and biochemistry investigations (hemoglobin, white cell count, glucose, urea and sodium). For each patient, the modified pre-stroke mRS, was ascertained from nursing and medical records by stroke specialist nurses. Since biochemistry data were electronically available after January 2003, we included patients admitted to the unit between January 2003 and June 2013. We included all cases of acute ischemic or hemorrhagic stroke, but excluded subarachnoid hemorrhage (SAH) and cases of recurrent stroke.

Admission serum sodium was used to classify participants into mild ( $\mathrm{Na} 130-134 \mathrm{mmol} / \mathrm{L})$, moderate (Na 125 $129 \mathrm{mmol} / \mathrm{L})$ and severe $(\mathrm{Na}<125 \mathrm{mmol} / \mathrm{L})$ hyponatremia, normonatremia ( $\mathrm{Na} 135-145 \mathrm{mmol} / / \mathrm{L})$ and hypernatremia $(\mathrm{Na}>145 \mathrm{mmol} / \mathrm{L})$. Follow-up was conducted from admission until December 2013 using the hospital administration system and validated using the NHS demographics system.

The statistical analysis was conducted using Stata 11.2/SE (College Station, TX, USA). One-way rank-based ANOVA or chi-squared tests compared baseline characteristics and mortality across sodium groups. A sequence of logistic regression models were then fitted to data to estimate the odds ratio of outcome (7 day mortality, death within 8-30 days, 1 year mortality) for each sodium group relative to the comparator group. The models were adjusted for a) age; b) age, sex, mRS and comorbidities (history of MI, Stroke, TIA, Dementia, Diabetes, PVD, heart failure, COPD and asthma); c) factors in (b) and stroke type and OSCP class; d) factors in (c) and glucose, urea, white cell count and hemoglobin. For mortality outcome at end of follow up, a Cox proportional 
hazards model was used along with Kaplan-Meier survival curves, with the same covariates as the model above, with censoring at the date of follow-up for those individuals still alive. A stratified analysis for those aged $<75$ years and those aged 75 or more was conducted using model (d) and a formal test of interaction was used to test the equality of the association with sodium group and outcome between these two age groups. 


\section{Results}

There were 9835 admissions during the study period. We excluded 99 individuals with no follow-up data, 156 individuals with SAH, 695 individuals with recurrent stroke and 494 individuals who had no available sodium results within one day of admission. Therefore, 8391 cases were included $(47.4 \%$ male, mean $( \pm$ SD) age $77.3( \pm$ 12.0) years).

The baseline point prevalence of hypernatremia was 3.3\%. The prevalences of mild, moderate and severe hyponatremia were $10.5 \%, 2.7 \%$ and $0.6 \%$, respectively. A comparison of baseline characteristics and outcomes according to natremic group is shown in Table 1 . The mortality for the entire cohort of 8391 patients was $12.9 \%$ at one week, $15.0 \%$ at 30 days and $39.1 \%$ at one year. Patients with sodium disturbances were older, had higher prior disability (depicted by pre-stroke mRS), higher white cell counts and higher prevalence of diabetes. Hyponatremia was more common in females whilst the prevalence of dementia was higher in hypernatremic patients.

Both hypo- and hypernatremia were associated with increased mortality at all selected time-points (Table 2 and Figure 1). However, the increased odds of death in mild hyponatremia (Na 130-134 mmol/L) and hypernatremia $(\mathrm{Na}>145 \mathrm{mmol} / \mathrm{L})$ did not reach statistical significance and were similar to the normonatremic group after adjustments.

The fully-adjusted sub-group analysis by age categories (Table 3) showed that hyponatremia in younger patients, especially when severe, was independently associated with higher mortality at all time-points. Intriguingly, younger patients with hyponatremia continued to have increased risk of death beyond one year after their stroke, even when hyponatremia was mild. In older patients, difference in odds of death by sodium level did not reach statistical significance at most time-points. 


\section{Discussion}

Our study is the largest to date to investigate the association between admission sodium levels and mortality after stroke. It confirms findings from smaller studies reporting that hyponatremia is an independent predictor of poor outcome after stroke both in the short [9] and long term [8]. Additionally, our findings show for the first time that this association is driven mostly by increased risk of death with hyponatremia in younger stroke patients. Also, the increased mortality with hypernatremia disappears after adjustment for potential confounders such as urea, a marker of hydration [16].

The $13.8 \%$ prevalence of hyponatremia on admission in our sample of stroke patients was similar to that observed in comparable literature by Rodrigues et al. (16\%) [9] and Huang et al. (12\%) [8], but lower than that seen in acute geriatric medicine wards (18\%) [17], where patients are older. However, mortality in our study was much higher than in the study by Huang, where mortality was just $8.8 \%$ at 3 years follow-up [8]. We also observed a higher mortality than the $29 \%$ seen at 1 year in the registry study by Rodrigues [9]. These differences are possibly due to our older, unselected sample population. It is unclear why our population is older than other published studies but probably reflects that frail older people in our study registry were not excluded from access to an acute stroke unit, as per best practice guidelines [18]. Also, our inclusion of both hemorrhagic and ischemic stroke, rather than just ischemic stroke, may have contributed to these differences.

Strikingly, younger patients with hyponatremia are at a higher risk of death. We initially hypothesised that older people would be at highest risk due to frailty and increased susceptibility to other adverse effects of hyponatremia, including confusion [12], falls [19] and fractures [20]. There are a number of possible explanations for this unexpected observation. Acute hyponatremia is well known to cause cerebral oedema [21] but older individuals may be more likely to survive because age-related cerebral atrophy will protect against fatal coning due to raised intracranial pressure. Also, the increased susceptibility to dyshomeostasis (and consequent hyponatremia) in older people [22] suggests that they may become hyponatremic due to less severe and/or more easily remediable causes than younger individuals. Such cases of hyponatremia, in older people, are predominantly mild and chronic (i.e. developing $>48$ hours) [11]. In this study we did not account for rate of development of hyponatremia which is a more important prognostic predictor than absolute serum sodium measurements [23]. Therefore, it is plausible that acute hyponatremia may be more common in younger people and that rate of development of hyponatremia may account for the worse prognosis in younger people with 
stroke. Since hyponatremia in younger patients continued to predict mortality beyond the short-term, it seems unlikely that cerebral oedema alone could continue to explain the association with increased mortality.

There were important differences at baseline (Table 1) between the various natremic groups, many of which may partly explain the unadjusted association between dysnatremias and death. In particular, older, frailer individuals with diabetes were more prone to dysnatremia, and those with hypernatremia were more likely to have dementia and present with total anterior circulation stroke. Hyponatremia was more common in women, in keeping with other observational studies [24].

Dysnatremia was associated with increased odds of death at all time-points, albeit with attenuation in magnitude of association after adjustment for multiple potential confounders. Surprisingly, the exception was that the odds of death with hypernatremia were significantly lower in the fully adjusted model. This suggests it is dehydration rather than hypernatremia that accounts for the excess mortality in the hypernatremic group. This is important as dehydration should be easily remediable, although stroke and hypernatremia may be terminal events for some individuals, and a palliative approach may have been appropriately instituted. The increased odds of death with mild hyponatremia were modest and did not reach statistical significance, but this may reflect a lack of power and would be unwise to dismiss since hyponatremia may also be very treatable. Previous groups have found that both mild hyponatremia and serum sodium levels within the lower range of normonatremia (serum sodium<137) are associated with increased mortality in hospitalised adult patients [25, 26].

Since hyponatremia is often chronic and notoriously challenging to manage [12], it is possible that many patients in this cohort remained chronically hyponatremic, as observed in non-stroke populations [27]. However, because we only utilised admission serum sodium measurements, this could not be assessed. Chronic hyponatremia in the general population is associated with increased mortality, even when mild [1], potentially explaining the longer term association with increased mortality in younger patients who have longer life expectancy compared to their older counterparts. Due to the limited life expectancy of our older co-morbid participants, it is possible that our analysis lacked power to detect an independent association with mortality at longer-term follow-up in the older patients due to the modest numbers of survivors and because moderate and severe hyponatremic cases were uncommon. There are no studies that carefully examine the causes of hyponatremia after stroke. Unfortunately, we do not have data on aetiology of hyponatremia encountered in this study either, which is a limitation of this report. 
Our study has some other limitations that should be considered. We used only baseline sodium measurements to highlight the potential impact of dysnatremia on stroke outcome. Also, in any observational study it is possible that there are unmeasured confounders. However, we robustly adjusted for patient related factors (demographics/co-morbidities/prestroke disability), stroke related factors (type), and acute haematological and biochemical parameters. We did not measure the severity of stroke other than by OCSP class. Despite these limitations, this report makes an important contribution to understanding of dysnatremia and post-stroke mortality. We studied a large and unselected study population and availability of outcome data was complete.

Whilst the association between hyponatraemia and mortality cannot prove causation, the independent association observed in this prospective study raises an important question. Would timely and appropriate management of hyponatraemia improve stroke mortality? This requires further study in a randomised controlled trial as it remains possible hyponatraemia is a marker of severity of underlying dyshomeostasis or disease. Our study does not provide information on the causes of hyponatremia, or what efforts were made to correct it. We therefore do not know how often hyponatremia was resistant to treatment.

In summary, our study provides the strongest and largest evidence to date on the association between admission dysnatraemias and mortality after stroke. This is clinically significant as it raises the possibility that better prevention and management of hypo- and hypernatremia may improve outcomes after stroke. Future studies should investigate the causes of hyponatremia after stroke, and whether therapeutic strategies to achieve normonatremia may lead to improved outcomes. 


\section{Acknowledgements}

We gratefully acknowledge the Stroke Services Data Team at Norfolk and Norwich University Hospital.

\section{Funding}

RLS is supported by a Career Research Fellowship from NHS Research Scotland. The Norfolk and Norwich Stroke \& TIA Register is maintained by the Norfolk \& Norwich Stroke Services. We obtained additional support from the NHS Research \& Development Research Capability Funding.

\section{Conflicts of interest}

The authors declare no conflicts of interest.

\section{Contributions}

RLS and PKM conceived the idea and designed the analysis plan with the input from ABC. JHB performed the record linkages. ABC analysed the data. PKM is the PI of the NNUH Stroke \& TIA register. RLS and KC drafted the manuscript. All authors contributed to the writing of the paper. 


\section{References}

[1] Gankam-Kengne F, Ayers C, Khera A, de Lemos J, Maalouf NM. Mild hyponatremia is associated with an increased risk of death in an ambulatory setting. Kidney Int. 2013;83:700-706

[2] Sturdik I, Adamcova M, Kollerova J, Koller T, Zelinkova Z, Payer J. Hyponatraemia is an independent predictor of in-hospital mortality. Eur J Intern Med. 2014;25:379-382

[3] Tzoulis P, Bagkeris E, Bouloux, PM. A case-control study of hyponatraemia as an independent risk factor for inpatient mortality. Clin. Endocrinol. 2014;81:401-407

[4] Hannon MJ, Thompson CJ. The syndrome of inappropriate antidiuretic hormone: prevalence, causes and consequences. Eur J Endocrinol. 2010;162:S5-S12

[5] Alam MN, Uddin MJ, Rahman KM et al. Electrolyte changes in stroke. Mymensingh Med J. 2012;21:584-9

[6] Wannamethee G, Whincup PH, Shaper AG, Lever AF. Serum sodium concentration and risk of stroke in middle-aged males. J Hypertens. 1994;2;971-9

[7] Lasek-Bal A, Holecki M, Kret B, Hawrot-Kawecka A, Dulawa J. Evaluation of Influence of Chronic Kidney Disease and Sodium Disturbances on Clinical Course of Acute and Sub-Acute Stage First-Ever Ischemic Stroke. Med Sci Monit. 2014;20:1389-94

[8] Huang WY1, Weng WC, Peng TI et al. Association of hyponatremia in acute stroke stage with three-year mortality in patients with first-ever ischemic stroke. Cerebrovasc Dis. 2012;34:55-62.

[9] Rodrigues B,Staff I, Fortunato G, McCullough LD. Hyponatremia in the prognosis of acute ischemic stroke. J Stroke Cerebrovasc Dis. 2014;23:850-4.

[10] Fofi L, Dall'armi V, Durastanti L et al. An observational study on electrolyte disorders in the acute phase of ischemic stroke and their prognostic value. J Clin Neurosci 2012;19:513-6

[11] Spasovski G, Vanholder R, Allolio B et al. Clinical practice guideline on diagnosis and treatment of hyponatraemia. Eur J Endocrinol. 2014;170:G1-G47.

[12] Soiza RL, Cumming K, Clarke JM, Wood KM, Myint PK. Hyponatremia: special considerations in older patients. J Clin Med. 2014;3:944-958 
[13] Bettencourt-Silva J, De La Iglesia B, Donell S, Rayward-Smith V. On creating a patient-centric database from multiple Hospital Information Systems. Methods Inf Med. 2012;51(3):210-20.

[14] Bonita R, Beaglehole R. Modification of Rankin Scale: Recovery of motor function after stroke. Stroke. $1988 ; 19: 1497-1500$

[15] Bamford J, Sandercock P, Dennis M, Burn J, Warlow C. Classification and natural history of clinically identifiable subtypes of cerebral infarction. Lancet. 1991;337:1521-1526

[16] Shepherd JI, Hatfield S, Kilpatrick ES. Is there still a role for measuring serum urea in an age of eGFR? Evidence of its use when assessing patient hydration. Nephron Clin Pract. 2009;113:c203-206

[17] Hoyle GE, Chua M, Soiza RL. Prevalence of hyponatremia in elderly patients. J Am Geriatr Soc. 2006; $54: 1473-4$

[18] Jauch EC, Saver JL, Adams HP et al. Guidelines for the early management of patients with acute ischemic stroke: a guideline for healthcare professionals from the American Heart Association/American Stroke Association. Stroke. 2013;44:870-947

[19] Renneboog B, Musch W, Vandemergel X, Manto MU, Decaux G. Mild Chronic Hyponatremia Is Associated With Falls, Unsteadiness, and Attention Deficits. Am J Med. 2006;119:71.e1-71.e8.

[20] Gankam-Kengne F, Andres C, Sattar L, Melot C, Decaux G. Mild hyponatremia and risk of fracture in the ambulatory elderly. QJM. 2008;101:583-588.

[21] Ayus JC, Achinger SG, Arieff A. Brain cell volume regulation in hyponatremia: role of sex, age, vasopressin, and hypoxia. Am J Physiol Ren Physiol. 2008;295:F619-F624

[22] Soiza RL, Hoyle GE, Chua MPW. Electrolyte and salt imbalance in older people: causes, management and implications. Rev Clin Gerontol. 2008;18:143-158

[23] Soiza RL, Talbot HSC. Management of hyponatremia in older people: old threats and new opportunities. Ther Adv Drug Safety. 2011; 9-17.

[24] Grikiniene J, Volbekas V, Stakisatis D. Gender differences of sodium metabolism and hyponatraemia as an adverse drug effect. Medicina. 2004;40:935-42 
[25] Wald RCM, Jaber BL, Price LL, Upadhyay A, Madias NE. Impact of Hospital-Associated Hyponatremia on Selected Outcomes. Arch Intern Med. 2010; 170(3):294-302.

[26] Waikar SS, Mount DB, Curhan GC. Mortality after hospitalization with mild, moderate, and severe hyponatremia. Am J Med. 2009; 122: 857-65.

[27] Cumming K, Hoyle GE, Hutchison JD, Soiza RL. Prevalence, Incidence and Etiology of Hyponatremia in Elderly Patients with Fragility Fractures. PLoS ONE. 2014; 9(2): e88272. 


\section{Tables}

Table 1: Sample characteristics categorised by sodium measurement $(\mathrm{mmol} / \mathrm{L})$

\begin{tabular}{|c|c|c|c|c|c|c|}
\hline Variable & Sodium $<125$ & Sodium 125-129 & Sodium 130-134 & Sodium 135-144 & Sodium $\geq 145$ & $\mathrm{P}$ value \\
\hline Number $(\mathrm{N})$ & 57 & 230 & 884 & 6945 & 275 & \\
\hline Age & $79.3(9.72)$ & $80.32(8.92)$ & $79.92(10.35)$ & $76.8(12.13)$ & $77.63(13.31)$ & $<0.001$ \\
\hline Male sex & $19(33.3)$ & $77(33.5)$ & $328(37.1)$ & $3413(49.1)$ & $138(50.2)$ & $<0.001$ \\
\hline Pre-stroke mRs* & & & & & & $<0.001$ \\
\hline 0 & $23(46.9)$ & $100(48.3)$ & $425(53.3)$ & $4286(66.3)$ & $119(49.4)$ & \\
\hline 1 & $8(16.3)$ & $35(16.9)$ & $108(13.5)$ & $680(10.5)$ & $19(7.9)$ & \\
\hline 2 & $5(10.2)$ & $20(9.7)$ & $84(10.5)$ & $501(7.7)$ & $16(6.6)$ & \\
\hline 3 & $9(18.4)$ & $28(13.5)$ & $104(13)$ & $564(8.7)$ & $43(17.8)$ & \\
\hline 4 & $2(4.1)$ & $16(7.7)$ & $48(6)$ & $313(4.8)$ & $23(9.5)$ & \\
\hline 5 & $2(4.1)$ & $8(3.9)$ & $29(3.6)$ & $121(1.9)$ & $21(8.7)$ & \\
\hline Previous stroke & $17(29.8)$ & $55(23.9)$ & $183(20.7)$ & 1319 (19) & $69(25.1)$ & 0.007 \\
\hline Previous TIA & $1(1.8)$ & $12(5.2)$ & $36(4.1)$ & $286(4.1)$ & $8(2.9)$ & 0.639 \\
\hline Stroke type & & & & & & 0.003 \\
\hline Bleed & $8(14)$ & $44(19.1)$ & $140(15.8)$ & 916 (13.2) & $24(8.7)$ & \\
\hline Infarct & $49(86)$ & $186(80.9)$ & $744(84.2)$ & $6029(86.8)$ & $251(91.3)$ & \\
\hline & & & & & & $<0.001$ \\
\hline Bamford Classification & & & & & & \\
\hline LACS $* *$ & $15(26.3)$ & $43(18.8)$ & $171(19.5)$ & $1597(23.1)$ & $48(17.5)$ & \\
\hline PACS $* * *$ & $14(24.6)$ & $74(32.3)$ & $278(31.7)$ & $2253(32.6)$ & $70(25.5)$ & \\
\hline POCS $* * * *$ & $12(21.1)$ & $43(18.8)$ & $151(17.2)$ & $1155(16.7)$ & $35(12.8)$ & \\
\hline $\mathrm{TACS} * * * * *$ & $10(17.5)$ & $42(18.3)$ & $196(22.3)$ & $1434(20.8)$ & $74(27)$ & \\
\hline $\mathrm{UNK} * * * * * *$ & $6(10.5)$ & $27(11.8)$ & $81(9.2)$ & $466(6.7)$ & $47(17.2)$ & \\
\hline & $725.81(1016.42)$ & $736(933.23)$ & $763.15(957.28)$ & & 895.94 & \\
\hline Follow-up period/ period till death & & & & $1029.05(1100.21)$ & $(1181.48)$ & $<0.001$ \\
\hline
\end{tabular}




\begin{tabular}{|c|c|c|c|c|c|c|}
\hline Glucose & $7.75(4.59)$ & $7.78(4.52)$ & $8.18(4.56)$ & $7.2(2.86)$ & $7.58(3.96)$ & 0.001 \\
\hline Urea & $7.31(6.19)$ & $7.29(5.55)$ & $7.8(5.01)$ & $7.69(4.12)$ & $13.95(11.02)$ & $<0.001$ \\
\hline Sodium & $120.18(4.34)$ & $127.55(1.35)$ & $132.53(1.37)$ & $139.37(2.32)$ & $147.69(4.67)$ & $<0.001$ \\
\hline HB & $12.8(2.12)$ & $12.8(1.81)$ & $12.89(2)$ & $13.58(1.9)$ & $13.6(2.35)$ & $<0.001$ \\
\hline WBC & $10.67(5.26)$ & $10.94(5.38)$ & $10.77(7.27)$ & $9.98(8.85)$ & $11.73(6)$ & $<0.001$ \\
\hline COPD & $8(14)$ & $14(6.1)$ & $60(6.8)$ & $393(5.7)$ & $17(6.2)$ & 0.065 \\
\hline $\mathrm{CHF}$ & $8(14)$ & $21(9.1)$ & $105(11.9)$ & $706(10.2)$ & $39(14.2)$ & 0.099 \\
\hline Diabetes & 11(19.3) & $34(14.8)$ & $131(14.8)$ & $762(11)$ & $36(13.1)$ & 0.001 \\
\hline Dementia & $1(1.8)$ & $15(6.5)$ & $39(4.4)$ & $249(3.6)$ & $29(10.5)$ & $<0.001$ \\
\hline Death 8-30 days & $7(12.3)$ & $26(11.3)$ & $105(11.9)$ & $618(8.9)$ & $44(16)$ & $<0.001$ \\
\hline Death within 1 year ${ }^{*}$ & $33(60)$ & $115(50.4)$ & $401(47.2)$ & 2491(37.4) & $148(54.8)$ & $<0.001$ \\
\hline Death & $40(70.2)$ & $156(67.8)$ & $553(62.6)$ & $3736(53.8)$ & $206(74.9)$ & $<0.001$ \\
\hline
\end{tabular}


Table 2: Odds Ratios (OR) for death at various time-points and Cox proportional hazards ratio (HR) for death using normonatremia as the reference category

\begin{tabular}{|c|c|c|c|c|c|}
\hline Variable & Sodium $<125$ & Sodium 125-129 & Sodium 130-134 & Sodium 135-144 & Sodium $\geq 145$ \\
\hline \multicolumn{6}{|c|}{7 day mortality $(\mathrm{OR})$} \\
\hline Model A* & $1.83(0.96-3.49)$ & $1.72(1.24-2.39)$ & $1.19(0.97-1.45)$ & 1 & $2.06(1.54-2.77)$ \\
\hline Model B** & $2.19(1.10-4.36)$ & $1.98(1.39-2.82)$ & $1.17(0.93-1.45)$ & 1 & $1.97(1.42-2.73)$ \\
\hline Model C*** & $2.84(1.29-6.22)$ & $2.03(1.35-3.08)$ & $1.01(0.79-1.30)$ & 1 & $1.51(1.04-2.19)$ \\
\hline Model D**** & $3.31(1.27-8.62)$ & $1.97(1.15-3.38)$ & $1.00(0.72-1.38)$ & 1 & $0.47(0.25-0.88)$ \\
\hline \multicolumn{6}{|c|}{ Death between 8-30 days (OR) } \\
\hline Model A* & $1.41(0.62-3.22)$ & $1.24(0.80-1.90)$ & $1.21(0.97-1.52)$ & 1 & $2.28(1.60-3.26)$ \\
\hline Model B** & $1.43(0.58-3.55)$ & $0.94(0.56-1.57)$ & $1.27(0.99-1.61)$ & 1 & $1.93(1.29-2.88)$ \\
\hline Model C*** & $1.66(0.64-4.34)$ & $1.04(0.61-1.78)$ & $1.24(0.96-1.60)$ & 1 & $1.73(1.12-2.67)$ \\
\hline Model D**** & $1.57(0.47-5.32)$ & $0.78(0.37-1.63)$ & $1.11(0.79-1.57)$ & 1 & $1.23(0.69-2.21)$ \\
\hline \multicolumn{6}{|c|}{ Death within 1 year $(\mathrm{OR})$} \\
\hline Model A* & $2.43(1.38-4.27)$ & $1.48(1.12-1.95)$ & $1.30(1.12-1.51)$ & 1 & $2.08(1.60-2.71)$ \\
\hline Model B** & $2.69(1.44-5.04)$ & $1.36(1.01-1.83)$ & $1.27(1.08-1.49)$ & 1 & $1.80(1.34-2.41)$ \\
\hline Model C*** & $3.23(1.66-6.30)$ & $1.37(1.00-1.89)$ & $1.23(1.03-1.48)$ & 1 & $1.61(1.16-2.23)$ \\
\hline Model D**** & $2.45(1.08-5.55)$ & $1.11(0.73-1.68)$ & $1.03(0.82-1.31)$ & 1 & $1.30(0.82-2.08)$ \\
\hline \multicolumn{6}{|l|}{ Death (HR) } \\
\hline Model A* & $1.39(1.02-1.91)$ & $1.30(1.11-1.53)$ & $1.16(1.06-1.27)$ & 1 & $1.68(1.45-1.94)$ \\
\hline Model B** & $1.46(1.05-2.04)$ & $1.26(1.06-1.50)$ & $1.14(1.03-1.26)$ & 1 & $1.55(1.33-1.81)$ \\
\hline Model C*** & $1.43(1.03-2.00)$ & $1.29(1.08-1.53)$ & $1.08(0.98-1.19)$ & 1 & $1.42(1.22-1.66)$ \\
\hline Model D**** & $1.67(1.13-2.47)$ & $1.20(0.96-1.51)$ & $1.05(0.92-1.19)$ & 1 & $1.10(0.88-1.36)$ \\
\hline
\end{tabular}

****model d- adjustment as for model c plus adjustment for haematological and biochemistry data 
Table 3: Fully-adjusted sub-group analysis by age categories

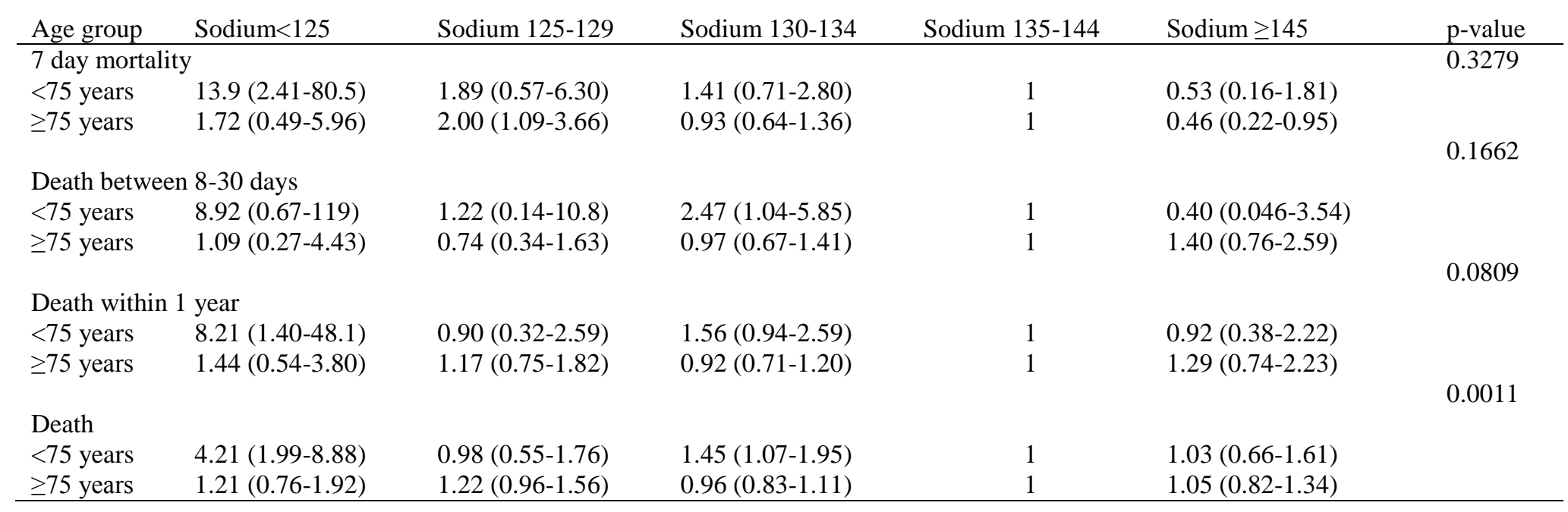


Figure 1: Kaplan Meier Survival Curves 


\section{Kaplan-Meier survival estimates}

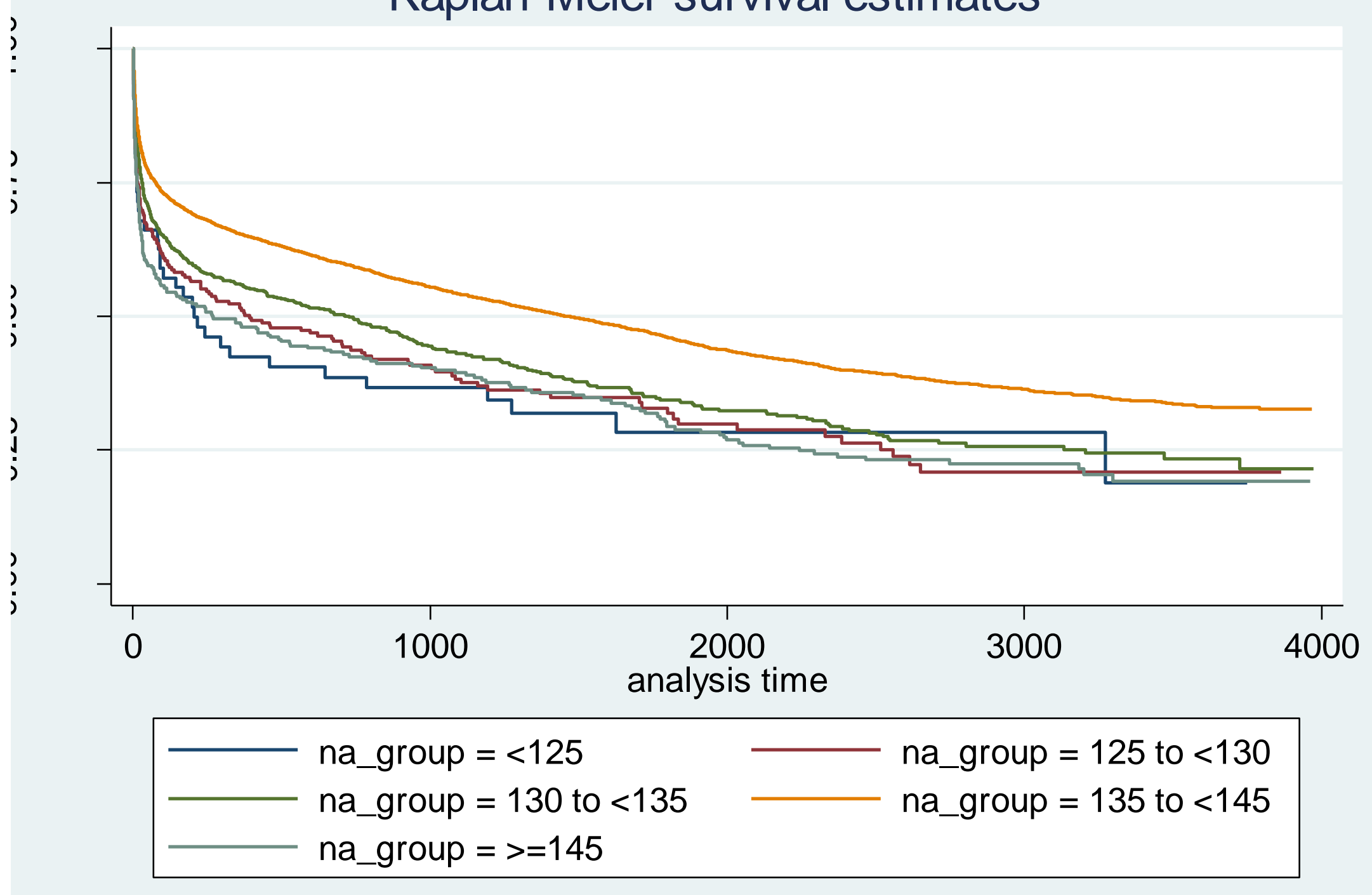

